\title{
Floquet Exponents and Bifurcations in Switched Converters
}

\author{
John Alexander Taborda, Fabiola Angulo and Gerard Olivar
}

Additional information is available at the end of the chapter

http://dx.doi.org/10.5772/51330

\section{Introduction}

Switched power converters are finding wide applications in the area of electrical energy conditioning. Many electronic devices have power converters to achieve high conversion efficiency and therefore low heat waste. Some of them are: drivers for industrial motion control, battery chargers, uninterruptible power supplies (UPS), electric vehicles, laptops, gadgets and mobile phones. Therefore control of power converters in order to optimize conversion efficiency is a current and challenging research topic. Pulsewidth modulation $(P W M)$ is the most used method to control power converters [11]-[12].

Digital-PWM controllers are a novel alternative to control power converters. These controllers have many advantages as programmability, high flexibility, reliability and easy implementation of advanced control algorithms. They can be designed with delays in the measured variables in order to guarantee the necessary computing time of the signal control. However, performance of PWM controllers is affected by delays.

In this chapter, we investigate the incidence of delays in a digital-PWM controller based on two novel techniques: Zero Average Dynamics (ZAD) and Fixed-Point Inducting Control (FPIC). Both control strategies have been developed, applied and widely analyzed in the last decade [5].

Floquet theory and smooth bifurcation theory can be used to define stability regions and to find optimum parameter sets (see for example [6]-[9]). In our case, three parameters should be tuned in the digital-PWM controller. Each parameter is denoted as: $k_{s}$ in ZAD strategy, $N$ in FPIC technique and $\tau$ is the number of delay periods in the measured variables [1].

The 3D-parameter space $\left(k_{s}, N, \tau\right)$ of the delayed PWM controller is analyzed and stability regions are bounded by Flip, Fold and Neimark-Sacker transitions. The presence of the three smooth bifurcations in the same nonlinear circuit is not common and this fact has not been reported widely in Digital-PWM switched converters. 
Stability, bifurcations and transient response of switched converters with Delayed PWM controllers can be studied more efficiently using an analysis of disturbances based on Floquet theory. We show that this procedure can be generalized to compute Floquet exponents for any number of delays $(\tau)$ in the control law $\left(d_{k}\right.$, so-called $d u t y$ cycle). We compare this approach with other methods which determine stability in switched converters. One of them is the computation of characteristic multipliers based on the jacobian matrix. Another one is the computation of Lyapunov exponents using a numeric routine. Each method gives equivalent information. However Floquet approach is the most appropriated when delays appear since this method does not require the evaluation of the jacobian matrix (its dimension increases when the number of delays is higher). The other two methods have this disadvantage [13].

The chapter is organized as follows. In Section 2 we present the general procedure to compute Floquet exponents in PWM switched converters. The particular case of a buck converter controlled with digital-PWM controller based on ZAD, FPIC and DELAY schemes, is presented in Section 3. The stability of fixed points in delayed PWM switched converters is discussed in Section 4, while fold, flip and Neimark-Sacker bifurcations are presented in Section 5. Finally, the conclusions and future work are presented in Section 6.

\section{Floquet-based procedure in PWM switched converters}

We assume that a PWM switched converter can be modelled as a piecewise-linear dynamical system, as it is written in equation (1). $\mathbf{x}$ is the state (nx1)-vector, $\mathbf{A}$ is the state (nxn)-matrix, $\mathbf{B}$ is the input $(n x 1)$-vector, $\mathbf{C}$ is the output $(1 x n)$-vector, $y$ is the scalar output and $u_{P W M}$ is the control signal.

$$
\left\{\begin{array}{l}
\dot{\mathbf{x}}=\mathbf{A} \mathbf{x}+\mathbf{B} u_{P W M} \\
y=\mathbf{C} \mathbf{x}
\end{array}\right.
$$

The PWM signal is a function of $d_{k}$ and the duty cycle is a function of the delayed state variables $\left(d_{k}=f(\mathbf{x}(k-\tau))\right)$. We consider the centered scheme given by equation (2).

$$
u_{P W M}(t)= \begin{cases}u_{l} & \text { if } k \leq t \leq k+d_{k} / 2 \\ u_{c} & \text { if } k+d_{k} / 2<t<k+1-d_{k} / 2 \\ u_{r} & \text { if } k+1-d_{k} / 2 \leq t \leq k+1\end{cases}
$$

First, we define the PWM Switched System including the discontinuity by using a unit step function $(\theta)$, as in equation (3) where $t \in\{0,(\tau+1) T\}, t_{s 1}=\left(\tau+d_{0} / 2\right) T, t_{s 2}=$ $\left(\tau+1-d_{0} / 2\right) T, \Delta u_{c l}=u_{c}-u_{l}$ and $\Delta u_{r c}=u_{r}-u_{c}$.

$$
\dot{\mathbf{x}}=\mathbf{A} \mathbf{x}+u_{l} \mathbf{B}+\Delta u_{c l} \theta\left(t-t_{s 1}\right) \mathbf{B}+\Delta u_{r c} \theta\left(t-t_{s 2}\right) \mathbf{B}
$$

According to Floquet theory we define, for the perturbed solution,

$$
\mathbf{x}=\mathbf{x}^{*}+e^{\mu T} \mathbf{p}(t)
$$

where

$\mu \in C$ is the so-called Floquet exponent and $\mathbf{p}(t+T)=\mathbf{p}(t)$ is an associated T-periodic function. 
Replacing the perturbed solution in equation (3) and neglecting the periodic solution, the variational equation of the system is obtained by equation (4) where $t_{s 1}^{*}=\left(\tau+\frac{d^{*}}{2}\right) T, t_{s 2}^{*}=$ $\left(\tau+1-\frac{d^{*}}{2}\right) T$ and $\mathbf{H}$ is a $(n x n)$-matrix which depends on the time differential related to the perturbation of the PWM signal. Matrix $\mathbf{H}$ depends on the control strategy.

$$
\dot{\mathbf{p}}=(\mathbf{A}-\mu \mathbf{I}) \mathbf{p}+\left(\Delta u_{r c} e^{-\mu\left(t-t_{s 2}^{*}\right)} \delta\left(t-t_{s 2}^{*}\right)-\Delta u_{c l} e^{-\mu\left(t-t_{s 1}^{*}\right)} \delta\left(t-t_{s 1}^{*}\right)\right) \mathbf{H} \mathbf{p}(0)
$$

The solution of the variational equation is stated in (5) where $z=e^{-\mu T}$.

$$
\mathbf{p}(1)=\left(z e^{\mathbf{A} T}+z^{\tau+1}\left(\Delta u_{r c} e^{\mathbf{A} T \frac{d^{*}}{2}}-\Delta u_{l c} e^{\mathbf{A} T\left(1-\frac{d^{*}}{2}\right)}\right) \mathbf{H}\right) \mathbf{p}(0)
$$

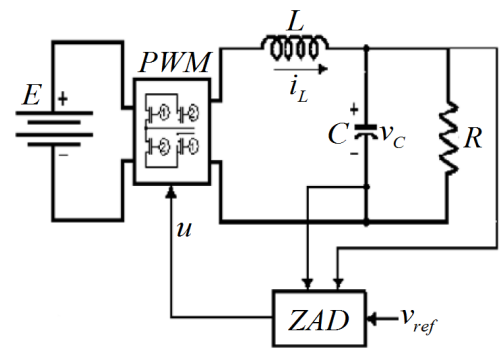

(a)

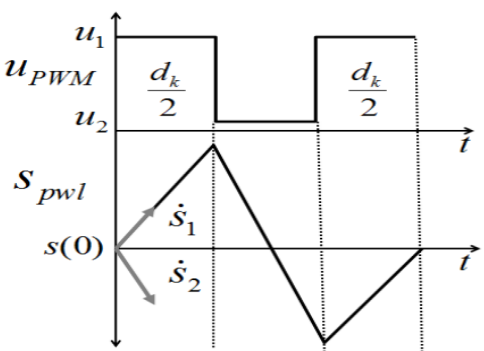

(b)

Figure 1. (a). Scheme of a PWM-controlled Buck converter with ZAD-strategy. (b). Piecewise-linear error dynamic $\left(s_{p w l}\right)$ in a sampling period.

\section{Floquet exponents in a synchronous buck converter}

The relevant roles of Floquet exponents on analysis, design and control of PWM switched converters are analyzed in a synchronous buck converter. Its main feature is that the output value $V_{o}$ is lower than the source $E$ (step down converter). Figure $1(a)$ shows a scheme of buck converter controlled with ZAD strategy.

The mathematical model for the synchronous buck converter can be written in compact form as:

$$
\left(\begin{array}{c}
\dot{x}_{1} \\
\dot{x}_{2}
\end{array}\right)=\left(\begin{array}{cc}
\frac{-1}{R C} & \frac{1}{C} \\
\frac{-1}{L} & \frac{-r_{L}}{L}
\end{array}\right)\left(\begin{array}{c}
x_{1} \\
x_{2}
\end{array}\right)+\left(\begin{array}{c}
0 \\
\frac{E}{L}
\end{array}\right) u_{P W M}
$$

where $x_{1}=v_{C}, x_{2}=i_{L}$ and $u_{P W M}$ belongs to the discrete set $\{-1,1\}$.

For the $Z A D$ condition, a piecewise-linear function is defined as equation (7). Figure $1(b)$ shows a scheme of $s_{p w l}$ in a period sampling.

$$
s_{p w l}(t)= \begin{cases}s_{1}+(t-k T) \dot{s}_{1} & \text { if } k T \leq t \leq k T+\frac{d_{k}}{2} \\ s_{2}+\left(t-k T+\frac{d_{k}}{2}\right) \dot{s}_{2} & \text { if } k T+\frac{d_{k}}{2}<t<k T+\left(T-\frac{d_{k}}{2}\right) \\ s_{3}+\left(t-k T+T+\frac{d_{k}}{2}\right) \dot{s}_{1} & \text { if } k T+\left(T-\frac{d_{k}}{2}\right) \leq t \leq(k+1) T\end{cases}
$$


where

$$
\begin{array}{lr}
\dot{s}_{1}=\left.\left(\dot{x}_{1}+k_{s} \ddot{x}_{1}\right)\right|_{x=x(k T), u=1} & \dot{s}_{2}=\left.\left(\dot{x}_{1}+k_{s} \ddot{x}_{1}\right)\right|_{x=x(k T), u=0} \\
s_{1}=\left.\left(x_{1}-r e f+k_{s} \dot{x}_{1}\right)\right|_{x=x(k T), u=1} s_{2}=\frac{d}{2} \dot{s}_{1}+s_{1} s_{3}=s_{1}+(T-d) \dot{s}_{2}
\end{array}
$$

and $k_{s}$ is a positive constant. Therefore, the zero average condition is

$$
\int_{k T}^{(k+1) T} s_{p w l}(t) d t=0
$$

Now, finding $d_{k}$ means solving equation (9) and redefining the duty cycle as $d_{z a d}$

$$
d_{z a d}=\frac{2 s_{1}+T \dot{s}_{2}}{\dot{s}_{2}-\dot{s}_{1}}
$$

The FPIC control law is given by equation (11), where $N$ is the control parameter and $d_{s s}$ is the duty cycle when the stationary state is reached.

$$
d_{k}=\frac{d_{z a d}+N d_{s s}}{N+1}
$$

This result can be expressed as a linear combination of the state variables, where $c_{1}, c_{2}$ and $c_{3}$ are constants.

$$
d_{k}=c_{1} x_{1}(k-\tau)+c_{2} x_{2}(k-\tau)+c_{3}
$$

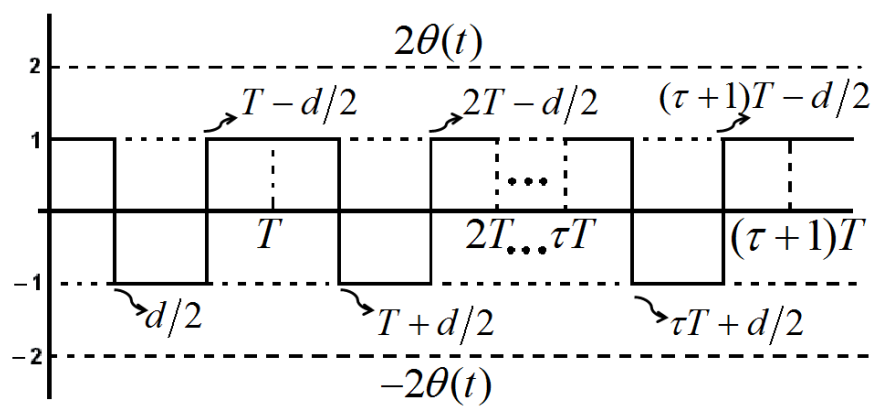

Figure 2. Scheme of centered PWM function depending on delays $(\tau)$.

Now, we should define the variational equation of the buck converter using the general procedure described in Section 2. Basically, we apply to the periodic solution ( $\left.\mathbf{x}^{*}\right)$ an appropriate perturbation using exponential functions $\left(e^{\mu t}\right)$. The stability of the digital-PWM power converter can be inferred studying the behavior of the perturbation. If the real part of the exponent $\mu$ is positive, the perturbation will tend to infinity and the solution will be unstable. If the real part of the exponent $\mu$ is negative, the perturbation will tend to zero and the solution will be stable. 
Therefore, we can find the variational equation based on the dynamical equations of the system. We use a more compact expression applying the following change of variables: $x_{1}=v_{C} / E, x_{2}=\frac{1}{E} \sqrt{\frac{L}{C}} i_{L}$ and $t=\tau / \sqrt{L C}$, thus $\gamma=\frac{1}{R} \sqrt{\frac{L}{C}}, \beta=r_{L} \sqrt{\frac{C}{L}}$ and the sampling period is $T=T_{\mathcal{C}} / \sqrt{L C}[10]$.

$$
\left(\begin{array}{c}
\dot{x_{1}} \\
\dot{x_{2}}
\end{array}\right)=\left(\begin{array}{cc}
-\gamma & 1 \\
-1 & -\beta
\end{array}\right)\left(\begin{array}{l}
x_{1} \\
x_{2}
\end{array}\right)+\left(\begin{array}{l}
0 \\
1
\end{array}\right) u_{P W M}
$$

We use the parameter values of an experimental prototype reported in [3]. We fix $R=20 \Omega$, $C=40 \mu \mathrm{F}, L=2 \mathrm{mH}, r_{L}=0 \Omega, v_{r e f}=32 \mathrm{~V}, E=40 \mathrm{~V}$ and the sampling period $T_{C}=50 \mu \mathrm{s}$. Therefore, the dimensionless parameters are $\gamma=0.35, \beta=0$ and $T=0.1767$.

For simplicity, in the remainder of Section 3, we note $d_{k}$ as $d$ with $d \in[0, T]$, and we present the procedure for one period sampling $t \in[\tau T,(\tau+1) T]$ (general notation was used in Section 2). The signal control $u_{P W M}$ is defined as equation (14), where $t_{s 1}=\left(\tau T+\frac{d}{2}\right)$ and $t_{s 2}=$ $\left((\tau+1) T-\frac{d}{2}\right)$.

$$
u_{P W M}=\left\{\begin{array}{cc}
1 & \tau T<t \leqslant t_{s 1} \\
-1 & t_{s 1}<t \leqslant t_{s 2} \\
1 & t_{s 2}<t \leqslant(\tau+1) T
\end{array}\right.
$$

Figure 2 illustrates the mechanism to model the centered pulse $\left(u_{P W M}\right)$ using unit step

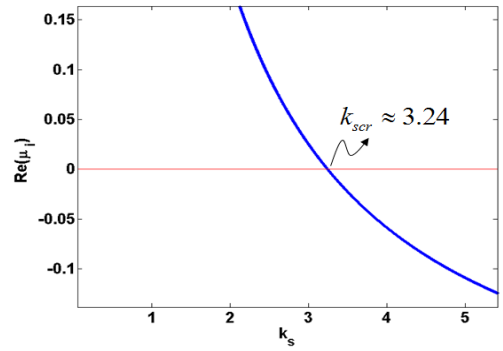

(a) $\tau=0, N=0$

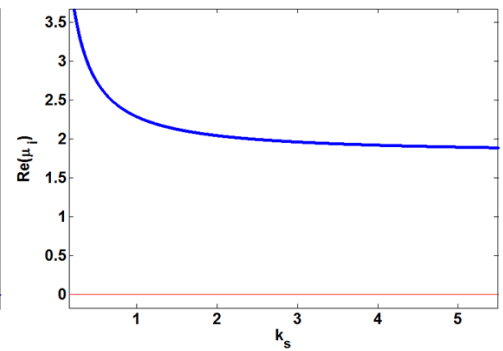

(b) $\tau=1, N=0$

Figure 3. The evolution of the real part of the Floquet exponents when $k_{s}$ is varied in the ZAD controller. $(a)$. without delay and without FPIC, $(b)$. with one delay and without FPIC

functions $\theta(t)$. The duty cycle depend on state variables in the instant $t=(k-\tau) T$. Equation (15) shows as two unit step functions can be used to model the centered pulse.

$$
\left(\begin{array}{c}
\dot{x_{1}} \\
\dot{x_{2}}
\end{array}\right)=\left(\begin{array}{cc}
-\gamma & 1 \\
-1 & -\beta
\end{array}\right)\left(\begin{array}{l}
x_{1} \\
x_{2}
\end{array}\right)+\left(\begin{array}{l}
0 \\
1
\end{array}\right)-2 \theta\left(t-t_{s 1}\right)\left(\begin{array}{l}
0 \\
1
\end{array}\right)+2 \theta\left(t-t_{s 2}\right)\left(\begin{array}{l}
0 \\
1
\end{array}\right)
$$

Equation (15) implies that duty cycle can be defined in function of state variables in an initial instant $(t=0)$ because the delay information was included in the unit step functions. 
Therefore, we can define $(d / 2)$ such as equation (16) where $c_{1}, c_{2}$ and $c_{3}$ are given by equations (17), (18) and (19), respectively.

$$
\begin{gathered}
\frac{d}{2}=c_{1} x_{1}(0)+c_{2} x_{2}(0)+c_{3} \\
c_{1}=\frac{2-\gamma\left(2 k_{s}+T\left(1-\gamma k_{s}\right)\right)-k_{s} T}{-4 k_{s}(N+1)} \\
c_{2}=\frac{2 k_{s}+T\left(1-k_{s}(\gamma+\beta)\right)}{-4 k_{s}(N+1)} \\
c_{3}=\frac{2 r e f+k_{s} T}{4 k_{s}}+\frac{N d_{s s}}{2(N+1)}
\end{gathered}
$$

Let the perturbed periodic orbit be

$$
x_{1}(t)=x_{1}^{*}(t)+e^{\mu t} p_{1}(t), \quad x_{2}(t)=x_{2}^{*}(t)+e^{\mu t} p_{2}(t),
$$

where the superstar labels are the periodic solutions and $e^{\mu t} p_{1}(t), e^{\mu t} p_{2}(t)$ are the perturbations. Then, we replace the perturbed periodic orbit in equation (15).

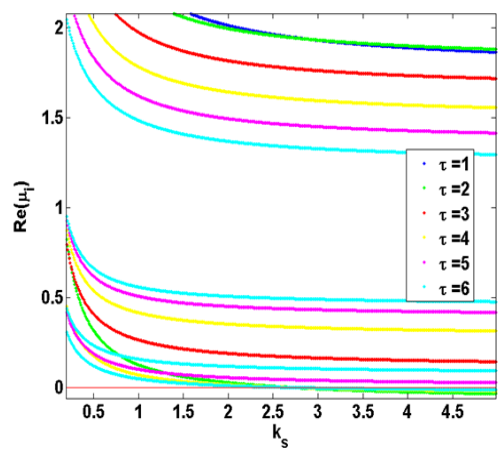

(a) ZAD scheme (without FPIC)

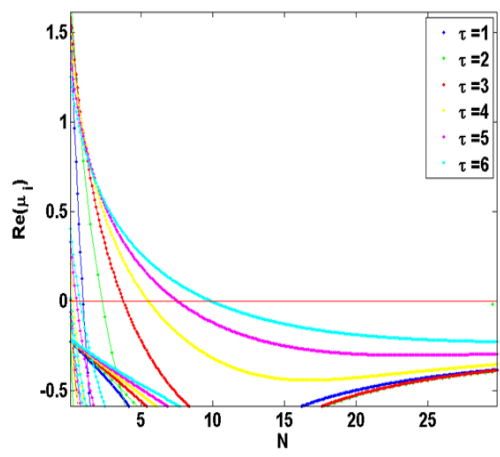

(b) ZAD-FPIC scheme

Figure 4. The evolution of the real part of the Floquet exponents for several delays in the Digital-PWM converter based on ZAD or ZAD-FPIC techniques. $(a) . k_{s}$ is varied between $[0 ; 5]$ with $N=0,(b)$. $N$ is varied between $[0 ; 30]$ with $k_{s}=4.5$.

$\dot{x}_{1}^{*}+\mu e^{\mu t} p_{1}+e^{\mu t} \dot{p}_{1}=-\gamma\left(x_{1}^{*}+e^{\mu t} p_{1}\right)+\left(x_{2}^{*}+e^{\mu t} p_{2}\right)$

$\dot{x}_{2}^{*}+\mu e^{\mu t} p_{2}+e^{\mu t} \dot{p}_{2}=-\left(x_{1}^{*}+e^{\mu t} p_{1}\right)-\beta\left(x_{2}^{*}+e^{\mu t} p_{2}\right)+1-2 \theta\left(t-\left(\tau T+\frac{d}{2}\right)\right)+$ $2 \theta\left(t-\left((\tau+1) T-\frac{d}{2}\right)\right)$

Unit step functions are replaced as follows. The periodic solution of duty cycle is noted:

$\frac{d^{*}}{2}=c_{1} x_{1}^{*}(0)+c_{2} x_{2}^{*}(0)+c_{3}$

then in the first unit step function,

$\theta\left(t-t_{s 1}^{*}\right)=\theta\left(t-\left(\tau T+\frac{d^{*}}{2}\right)\right)=\theta\left(t-\left(\tau T+c_{1} x_{1}^{*}(0)+c_{2} x_{2}^{*}(0)+c_{3}\right)\right)$ 
therefore, the step function in function of the perturbed periodic solution is

$\theta\left(t-\left(\tau T+\frac{d}{2}\right)\right)=\theta\left(t-\left(\tau T+c_{1}\left(x_{1}^{*}(0)+p_{1}(0)\right)+c_{2}\left(x_{2}^{*}(0)+p_{2}(0)\right)+c_{3}\right)\right)$

computing a first order Taylor expansion approximation of the unity step function, we obtain $\theta\left(t-\left(\tau T+\frac{d}{2}\right)\right)=\theta\left(t-\left(\tau T+\frac{d^{*}}{2}\right)\right)-\delta\left(t-\left(\tau T+\frac{d^{*}}{2}\right)\right)\left(c_{1} p_{1}(0)+c_{2} p_{2}(0)\right)$

where $\delta(t)$ is the Dirac delta function with $\delta(t)=\frac{d \theta(t)}{d t}$. The same considerations are applied in the second unity step function $\theta\left(t-t_{s 2}\right)$. The dynamic of the perturbed periodic solution is

$$
\begin{gathered}
\dot{x}_{1}^{*}+\mu e^{\mu t} p_{1}+e^{\mu t} \dot{p}_{1}=-\gamma x_{1}^{*}-\gamma e^{\mu t} p_{1}+x_{2}^{*}+e^{\mu t} p_{2} \\
\dot{x}_{2}^{*}+\mu e^{\mu t} p_{2}+e^{\mu t} \dot{p}_{2}=-x_{1}^{*}-e^{\mu t} p_{1}-\beta x_{2}^{*}-\beta e^{\mu t} p_{2}+1-2 \theta\left(t-\left(\tau T+\frac{d^{*}}{2}\right)\right)+ \\
2 \delta\left(t-\left(\tau T+\frac{d^{*}}{2}\right)\right)\left(c_{1} p_{1}(0)+c_{2} p_{2}(0)\right)+2 \theta\left(t-\left((\tau+1) T-\frac{d^{*}}{2}\right)\right) \\
+2 \delta\left(t-\left((\tau+1) T-\frac{d^{*}}{2}\right)\right)\left(c_{1} p_{1}(0)+c_{2} p_{2}(0)\right)
\end{gathered}
$$

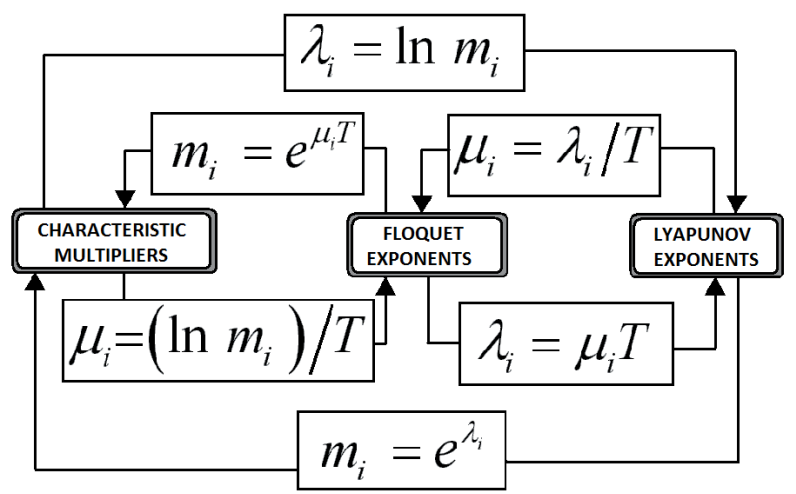

Figure 5. Scheme of equivalent transformations between Floquet exponents $\left(\mu_{i}\right)$, Lyapunov exponents $\left(\lambda_{i}\right)$ and characteristics multipliers $\left(m_{i}\right)$.

Neglecting the periodic solution $\mathbf{x}^{*}$, we obtain the dynamic of the perturbation.

$$
\begin{gathered}
\mu e^{\mu t} p_{1}+e^{\mu t} \dot{p}_{1}=-\gamma e^{\mu t} p_{1}+e^{\mu t} p_{2} \\
\mu e^{\mu t} p_{2}+e^{\mu t} \dot{p}_{2}=-e^{\mu t} p_{1}-\beta e^{\mu t} p_{2}+2 \delta\left(t-\left(\tau T+\frac{d^{*}}{2}\right)\right)\left(c_{1} p_{1}(0)+c_{2} p_{2}(0)\right)+ \\
2 \delta\left(t-\left((\tau+1) T-\frac{d^{*}}{2}\right)\right)\left(c_{1} p_{1}(0)+c_{2} p_{2}(0)\right)
\end{gathered}
$$

multiplying both sides of the equation by $e^{-\mu t}$, we obtain:

$$
\dot{p}_{1}=-\mu p_{1}-\gamma p_{1}+p_{2}
$$




$$
\begin{gathered}
\dot{p}_{2}=-p_{1}-\mu p_{2}-\beta p_{2}+2 e^{-\mu t} \delta\left(t-\left(\tau T+\frac{d^{*}}{2}\right)\right)\left(c_{1} p_{1}(0)+c_{2} p_{2}(0)\right)+ \\
2 e^{-\mu t} \delta\left(t-\left((\tau+1) T-\frac{d^{*}}{2}\right)\right)\left(c_{1} p_{1}(0)+c_{2} p_{2}(0)\right)
\end{gathered}
$$

Simplifying the expressions and writing in matrix notation we obtain the variational equation of the buck converter. Note that the terms $e^{-\mu t}$ only have sense in $t=\vartheta$ when these are multiplied by Dirac delta functions $\delta(t-\vartheta)$.

$$
\dot{\mathbf{p}}=\left(\begin{array}{cc}
-\gamma-\mu & 1 \\
-1 & -\beta-\mu
\end{array}\right) \mathbf{p}+e^{-\mu t_{s 1}^{*}} \delta\left(t-t_{s 1}^{*}\right) \mathbf{H} \mathbf{p}(0)+e^{-\mu t_{s 2}^{*}} \delta\left(t-t_{s 2}^{*}\right) \mathbf{H p}(0)
$$

Therefore, equation (20) is the variational equation where $t_{s 1}^{*}=\tau T+\frac{d^{*}}{2}, t_{s 2}^{*}=(\tau+1) T-\frac{d^{*}}{2}$ and,

$$
\mathbf{H}=\left(\begin{array}{cc}
0 & 0 \\
2 c_{1} & 2 c_{2}
\end{array}\right), \dot{\mathbf{p}}=\left(\begin{array}{c}
\dot{p}_{1} \\
\dot{p}_{2}
\end{array}\right), \mathbf{p}=\left(\begin{array}{c}
p_{1} \\
p_{2}
\end{array}\right), \mathbf{a}_{e}(0)=\left(\begin{array}{c}
p_{1}(0) \\
p_{2}(0)
\end{array}\right)
$$

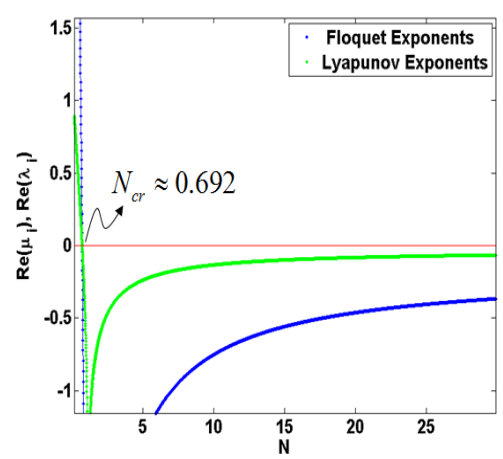

(a) $\tau=0, k_{s}=0.125$

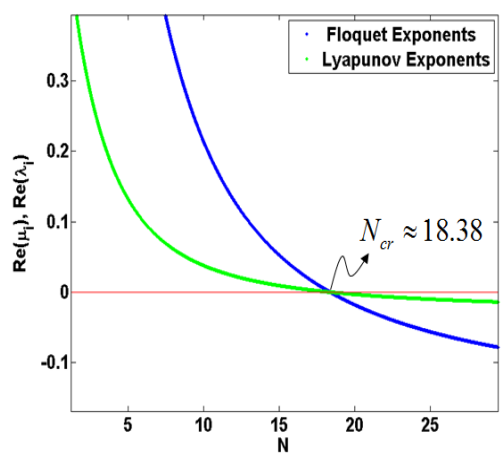

(b) $\tau=1, k_{\mathrm{s}}=0.125$

Figure 6. The evolution of the real part of the Floquet and Lyapunov exponents when $N$ is varied. (a). without delay, $(b)$. with one delay.

Note that equation (20) can be written in a compact form as:

$$
\dot{\mathbf{p}}=\mathbf{M} \mathbf{p}+e^{-\mu t}\left[\delta\left(t-\left(\tau T+\frac{d}{2}\right)\right)+\delta\left(t-(\tau+1) T+\frac{d}{2}\right)\right] \mathbf{H} \mathbf{p}(0)
$$

with $\mathbf{M}$ and $\mathbf{H}$ defined according to the equation (20), and again, for simplicity we note $d=$ $d^{*}$. For solving this piecewise-smooth ordinary differential equation, we write $z=e^{-\mu T}$, $\mathbf{M}=\mathbf{M}_{1}+\mathbf{M}_{2}$ with $e^{\mathbf{M} t}=e^{\mathbf{M}_{1} t} e^{\mathbf{M}_{2} t}$.

$$
\begin{gathered}
\mathbf{M}_{1}=\left(\begin{array}{cc}
-\frac{\gamma}{2}-\frac{\beta}{2}-\mu & 0 \\
0 & -\frac{\gamma}{2}-\frac{\beta}{2}-\mu
\end{array}\right) \\
\mathbf{M}_{2}=\left(\begin{array}{cc}
-\frac{\gamma}{2}+\frac{\beta}{2} & 1 \\
-1 & \frac{\gamma}{2}-\frac{\beta}{2}
\end{array}\right)
\end{gathered}
$$


The particular selection of $\mathbf{M}_{1}$ and $\mathbf{M}_{2}$ allows a easier solution of state-transition matrix $e^{\mathbf{M} t}$. The first exponential matrix $e^{\mathbf{M}_{1} t}$ is computed using the identity matrix, while the second exponential matrix $e^{\mathbf{M}_{2} t}$ is computed using sine and cosine functions.

$$
\begin{gathered}
e^{\mathbf{M}_{1} t}=e^{-\left(\frac{\gamma}{2}+\frac{\beta}{2}+\mu\right) t} \mathbf{I} \\
e^{\mathbf{M}_{2} t}=\left(\begin{array}{cc}
-\frac{\alpha_{1}}{\alpha_{2}} \operatorname{sen}\left(\alpha_{2} t\right)+\cos \left(\alpha_{2} t\right) & \frac{1}{\alpha_{2}} \operatorname{sen}\left(\alpha_{2} t\right) \\
-\frac{1}{\alpha_{2}} \operatorname{sen}\left(\alpha_{2} t\right) & \frac{\alpha_{1}}{\alpha_{2}} \operatorname{sen}\left(\alpha_{2} t\right)+\cos \left(\alpha_{2} t\right)
\end{array}\right)
\end{gathered}
$$

where $\alpha_{1}=\left(\frac{\gamma}{2}-\frac{\beta}{2}\right)$ and $\alpha_{2}=\sqrt{1-\alpha_{1}^{2}}$.

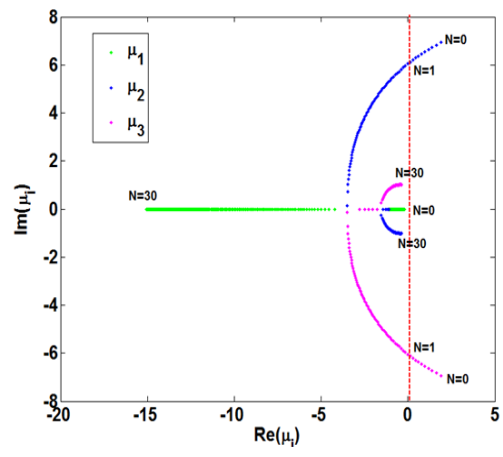

(a)

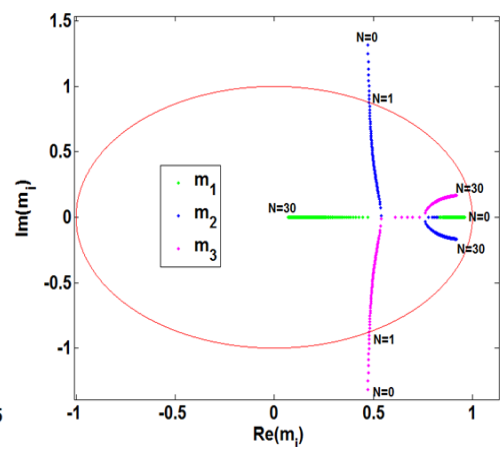

(b)

Figure 7. Locus of Floquet exponents and characteristic multipliers when $N$ is varied between $[0 ; 30]$ for $\tau=1$. (a). Floquet locus, $(b)$. Characteristic multipliers locus.

The piecewise-smooth ordinary differential equation can be solved in each interval with special attention in the discontinuities due to Dirac delta functions.

1). Initially, we compute the solution between $t=0$ and $t=\frac{d}{2}$

$$
\mathbf{p}\left(\frac{d}{2}\right)_{-}=e^{\mathbf{M}_{1} \frac{d}{2}} e^{\mathbf{M}_{2} \frac{d}{2}} \mathbf{p}(0)
$$

then we compute $\mathbf{p}\left(\frac{d}{2}\right)_{+}$integrating about the discontinuity as follows.

$$
\begin{gathered}
\mathbf{p}\left(\frac{d}{2}\right)_{+}=\mathbf{p}\left(\frac{d}{2}\right)_{-}+e^{-\mu\left(\tau T+\frac{d}{2}\right)} \mathbf{H} \mathbf{p} \\
\mathbf{p}\left(\frac{d}{2}\right)_{+}=\left(e^{\mathbf{M}_{1} \frac{d}{2}} e^{\mathbf{M}_{2} \frac{d}{2}}+e^{-\mu\left(\tau T+\frac{d}{2}\right)} \mathbf{H}\right) \mathbf{p}(0)
\end{gathered}
$$

2). Now, we compute the solution in the interval between $t=\left(\frac{d}{2}\right)$ and $t=\left(T-\frac{d}{2}\right)$. 


$$
\begin{gathered}
\mathbf{p}\left(T-\frac{d}{2}\right)_{-}=e^{\mathbf{M}_{1}(T-d)} e^{\mathbf{M}_{2}(T-d)} \mathbf{p}\left(\frac{d}{2}\right)_{+} \\
\mathbf{p}\left(T-\frac{d}{2}\right)_{-}=e^{\mathbf{M}_{1}(T-d)} e^{\mathbf{M}_{2}(T-d)}\left(e^{\mathbf{M}_{1} \frac{d}{2}} e^{\mathbf{M}_{2} \frac{d}{2}}+e^{-\mu\left(\tau T+\frac{d}{2}\right)} \mathbf{H}\right) \mathbf{p}(0)
\end{gathered}
$$

in the discontinuity,

$$
\begin{gathered}
\mathbf{p}\left(T-\frac{d}{2}\right)_{+}=\mathbf{p}\left(T-\frac{d}{2}\right)_{-}+e^{-\mu\left((\tau+1) T-\frac{d}{2}\right)} \mathbf{H} \mathbf{p}(0) \\
\mathbf{p}\left(T-\frac{d}{2}\right)_{+}=\left(e^{\mathbf{M}_{1}(T-d)} e^{\mathbf{M}_{2}(T-d)} e^{\mathbf{M}_{1} \frac{d}{2}} e^{\mathbf{M}_{2} \frac{d}{2}}+e^{\mathbf{M}_{1}(T-d)} e^{\mathbf{M}_{2}(T-d)} e^{-\mu\left((\tau+1) T-\frac{d}{2}\right)} \mathbf{H}\right) \mathbf{p}(0)
\end{gathered}
$$

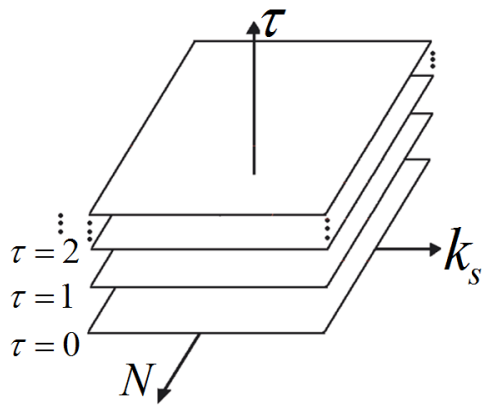

(a)

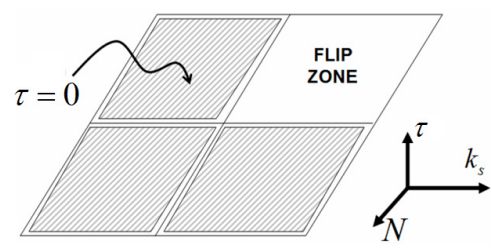

(b)

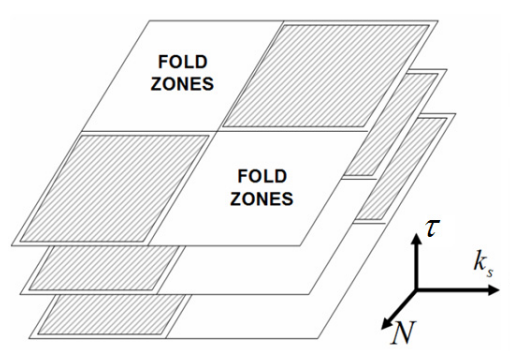

(c)

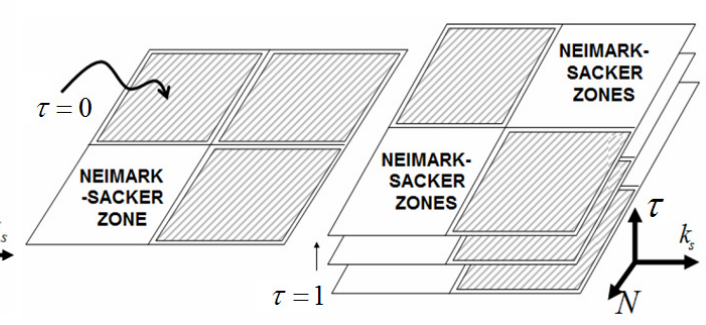

(d)

Figure 8. 3D parameter space of Digital-PWM switched converter and bifurcation zones. $(a) .\left(k_{s}, N, \tau\right)$ space where the lines $k_{s}=0$ and $N=-1$ separate each plane in four sub-spaces. ( $b$ ). flip bifurcation zone. $(c)$. fold bifurcation zones. $(d)$. Neimark-Sacker bifurcation zones.

3). Finally, we compute the solution in the last interval between $t=\left(T-\frac{d}{2}\right)$ and $T$.

$$
\begin{gathered}
\mathbf{p}(T)=e^{\mathbf{M}_{1} \frac{d}{2}} e^{\mathbf{M}_{2} \frac{d}{2}} \mathbf{p}\left(T-\frac{d}{2}\right)_{+} \\
\mathbf{p}\left(T-\frac{d}{2}\right)_{+}=\left(e^{\mathbf{M}_{1}(T-d)} e^{\mathbf{M}_{2}(T-d)} e^{\mathbf{M}_{1} \frac{d}{2}} e^{\mathbf{M}_{2} \frac{d}{2}}+\right.
\end{gathered}
$$




$$
\left.e^{\mathbf{M}_{1}(T-d)} e^{\mathbf{M}_{2}(T-d)} e^{-\mu\left((\tau+1) T-\frac{d}{2}\right)} \mathbf{H}\right) \mathbf{p}(0)
$$

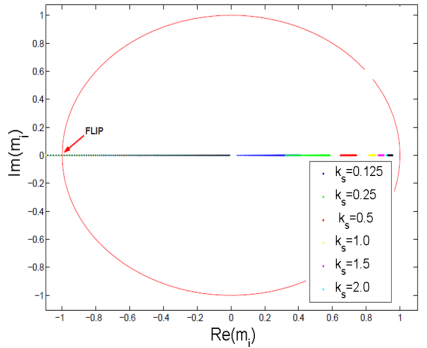

(a)

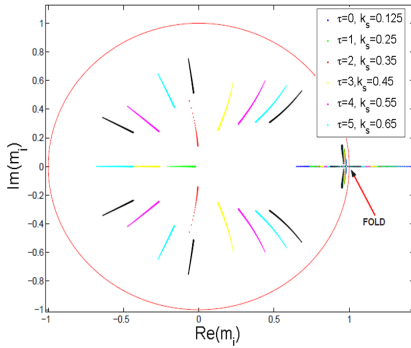

(b)

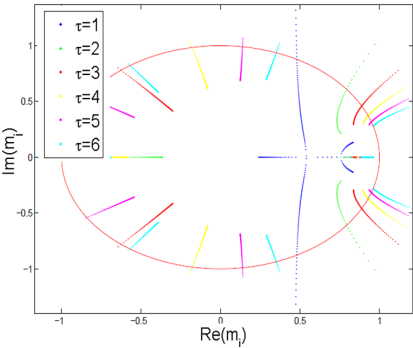

(c)

Figure 9. Evolution of characteristic multipliers in several bifurcation zones. (a). flip bifurcation zone $\left(\tau=0, k_{s}>0 N>-1\right)$. (b). fold bifurcation zones $\left(\tau>0, k_{s}>0 N<-1\right)$. (c). Neimark-Sacker bifurcation zones $\left(\tau>1, k_{s}>0 N>-1\right)$.

Equation (24) is the solution of the variational system where $z=e^{-\mu T}$.

$$
\mathbf{p}(T)=\mathbf{Q}_{1} \mathbf{p}_{e}(0)
$$

The matrix $\mathbf{Q}_{1}$ is given by equation (25), where $\alpha_{3}=\frac{\gamma}{2}+\frac{\beta}{2}$.

$$
\mathbf{Q}_{1}=\left(z e^{-\alpha_{3} T} e^{\mathbf{M}_{2} T}+z^{(\tau+1)} e^{-\alpha_{3}\left(T-\frac{d}{2}\right)} e^{\mathbf{M}_{2}\left(T-\frac{d}{2}\right)} \mathbf{H}+z^{(\tau+1)} e^{-\alpha_{3}\left(\frac{d}{2}\right)} e^{\mathbf{M}_{2}\left(\frac{d}{2}\right)} \mathbf{H}\right)
$$

The existence of T-periodic solution, i.e. $\mathbf{p}(T)=\mathbf{p}(0)$, depends on equation (26) is satisfied.

$$
\left(\mathbf{Q}_{1}-I\right) \mathbf{p}(0)=\mathbf{0}
$$

The stability of the periodic solution depends on whether the real part of each Floquet exponent is negative or not. If $\operatorname{det}\left(\mathbf{Q}_{1}-\mathbf{I}\right)=0$ implies $\mu$ negative.

We fix the parameter values in $\gamma=0.35 ; \beta=0 ; T=0.1767 ; k_{s}=4.5 ; N=0$ and $r e f=0.8$. Equation (27) shows equation (26) in function of the number of delays $(\tau)$. The equation is a polynomial in $z$ of degree $2(\tau+1)$.

$$
0.1 e-20 z^{2(\tau+1)}-1.867 z^{(\tau+2)}+1.911 z^{(\tau+1)}+0.93 z^{2}-1.899 z+1=0
$$

Assuming real-time behavior, i.e. $\tau=0$, the determinant is the second order polynomial of equation (28). The evolution of the real part of the Floquet exponents as parameter $k_{s}$ varies is displayed in figure 3(a). The T-periodic solution is stable for $k_{s}>3.24$.

$$
\left(\frac{1 e-10}{k_{s}^{2}}-\frac{0.00215387}{k_{s}}-0.9466771\right) z^{2}+\left(\frac{0.3443753}{k_{s}}-0.0535075\right) z+1=0
$$




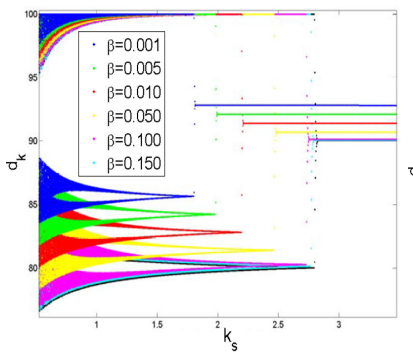

(a)

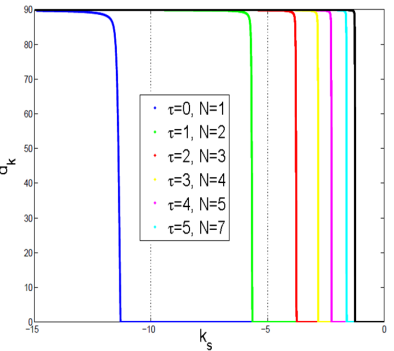

(b)

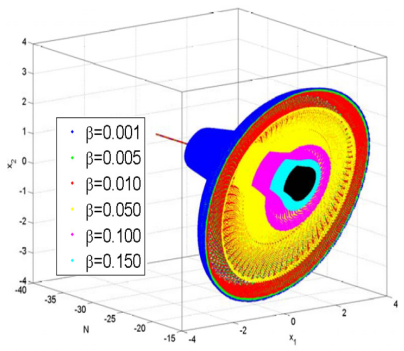

(c)

Figure 10. Examples of bifurcation diagrams in each zone. (a). flip bifurcation diagram $\left(\tau=0, k_{s}>0\right.$ $N>-1)$. (b). fold bifurcation diagram $\left(\tau>0, k_{s}>0 N<-1\right)$. (c). Neimark-Sacker bifurcation $\operatorname{diagram}\left(\tau>1, k_{s}>0 N>-1\right)$.

Assuming one-delay period, i.e. $\tau=1$, the determinant is the forth order polynomial of equation (29). The evolution of the real part of the Floquet exponents as parameter $k_{s}$ varies is displayed in figure $3(b)$. In this case, one Floquet exponent has positive real part for any $k_{s}$. Therefore, ZAD strategy should be combined with FPIC $(N \neq 0)$ to reach stable solutions.

$$
\frac{1 e-9}{k_{s}^{2}} z^{4}-\left(1.8867+\frac{0.002154}{k_{s}}\right) z^{3}+\left(\frac{0.344375}{k_{s}}+2.79635\right) z^{2}-1.90983 z+1=0
$$

For $\tau>1$, ZAD strategy is not sufficient to stabilize 1T-periodic orbit and ZAD-FPIC scheme

\begin{tabular}{|c|c|}
\hline Control Parameters & Stability Limit \\
\hline$(\tau=0),\left(k_{s}=4.5\right)$ & $N_{c r} \approx 0$ \\
\hline$(\tau=1),\left(k_{s}=4.5\right)$ & $N_{c r} \approx 0.99$ \\
\hline$(\tau=2),\left(k_{s}=4.5\right)$ & $N_{c r} \approx 2.32$ \\
\hline$(\tau=3),\left(k_{s}=4.5\right)$ & $N_{c r} \approx 3.79$ \\
\hline$(\tau=4),\left(k_{s}=4.5\right)$ & $N_{c r} \approx 5.53$ \\
\hline$(\tau=5),\left(k_{s}=4.5\right)$ & $N_{c r} \approx 7.55$ \\
\hline$(\tau=6),\left(k_{s}=4.5\right)$ & $N_{c r} \approx 9.89$ \\
\hline
\end{tabular}

Table 1. Critical value of stability $\left(N_{c r}\right)$ of buck converter controlled with ZAD-FPIC with several delay numbers. Figure $4(b)$ shows the evolution of real part of Floquet exponents.

is necessary. Figure $4(a)$ shows the evolution of Floquet exponents when $k_{s}$ is varied for several delay numbers and $N=0$. The number of Floquet exponents with positive real part increases as the delay number grows.

Figure $4(b)$ shows the results of ZAD-FPIC scheme when $k_{s}=4.5$ and $N$ is varied between $[0 ; 30]$. The critical value of stability $\left(N_{c r}\right)$ increases as the delay number grows. Table 1 summarizes the behavior of critical value for different delays.

The behavior of the critical value is similar when $N$ is fixed and $k_{s}$ is varied for several delay numbers. The value $k_{s c r}$ increases as the delay number grows. Table 2 shows this condition. 


\begin{tabular}{|c|c|}
\hline Control Parameters & Stability Limit \\
\hline$(\tau=0, N=0)$ & $k_{s} \approx 3.24$ \\
\hline$(\tau=1, N=2)$ & $k_{s} \approx 0.46$ \\
\hline$(\tau=2, N=3)$ & $k_{s} \approx 1.19$ \\
\hline$(\tau=3, N=4)$ & $k_{s} \approx 2.99$ \\
\hline$(\tau=4, N=6)$ & $k_{s} \approx 2.72$ \\
\hline$(\tau=5, N=8)$ & $k_{s} \approx 3.25$ \\
\hline$(\tau=6, N=10)$ & $k_{s} \approx 4.21$ \\
\hline
\end{tabular}

Table 2. Critical value of stability $\left(k_{s c r}\right)$ of buck converter controlled with ZAD-FPIC with several delay numbers. $(\tau=0$ to $\tau=6)$

\section{Stability of fixed points in delayed PWM switched converters}

In previous section, we show that the procedure based on variational equation can be used to compute Floquet exponents for any number of delays $(\tau)$. In this section, we compare this approach with other methods which determine stability in switched converters. One of them is the computation of characteristic multipliers based on the jacobian matrix. Another one is the computation of Lyapunov exponents using a numeric routine. Each method gives equivalent information. However Floquet approach is the most appropriated when delays appear since this method does not require the evaluation of the jacobian matrix (its dimension increases when the number of delays is higher). The other two methods have this disadvantage [13].

\subsection{Stability of 1-periodic orbit using Jacobian matrix}

The evaluation of the jacobian matrix is necessary to compute characteristic multipliers and Lyapunov exponents in PWM switched converters. The dimension of the jacobian matrix depends on the delay number considered in the control law. The order of Jacobian matrix is $2(\tau+1)$.

Poincaré map of the PWM switched converter can be used to determine the stability of 1-periodic orbit. Equation (30) presents the Poincaré map of synchronous buck converter with centered PWM control.

$$
\mathbf{x}((k+1) T)=e^{\mathbf{A} T} \mathbf{x}(k T)+\left(e^{\mathbf{A}\left(T-d_{k} / 2\right)}+\mathbf{I}\right) \mathbf{A}^{-1}\left(e^{\mathbf{A} d_{k} / 2}-\mathbf{I}\right) \mathbf{B}-e^{\mathbf{A} d_{k} / 2} \mathbf{A}^{-1}\left(e^{\mathbf{A}\left(T-d_{k}\right)}-\mathbf{I}\right) \mathbf{B}
$$

Real-time control law implies that the duty cycle $d_{k}$ depends on state variables in the instant $k T$, i.e., $d_{k}=c_{1} x_{1}(k T)+c_{2} x_{2}(k T)+c_{3}$. Therefore, Poincaré map (30) can be written as follows.

$$
\begin{aligned}
& x_{1}((k+1) T)=f_{1}\left(x_{1}(k T), x_{2}(k T)\right) \\
& x_{2}((k+1) T)=f_{2}\left(x_{1}(k T), x_{2}(k T)\right)
\end{aligned}
$$

Jacobian matrix of the system with $\tau=0$ can be computed with equation (32).

$$
\mathbf{A}_{n 0}=\left[\begin{array}{cc}
\frac{\partial f_{1}}{\partial x_{1}(k T)} & \frac{\partial f_{1}}{\partial x_{2}(k T)} \\
\frac{\partial f_{2}}{\partial x_{1}(k T)} & \frac{\partial f_{2}}{\partial x_{2}(k T)}
\end{array}\right]
$$


The matrix $\mathbf{A}_{n 0}$ should be evaluated in the fixed point $\left(\mathbf{A}_{n 0}=\left(\frac{\partial \mathbf{f}}{\partial \mathbf{x}_{i}}\right)_{\text {F.P. }}\right)$. In this case, we define F.P. as (ref, rref). Its eigenvalues (or characteristic multipliers) determine stability properties of the fixed point. The 1-periodic orbit is asymptotically stable if all characteristic multipliers have magnitude less than one $\left(\left|m_{i}\right|<1\right)$; it is unstable if at least one eigenvalue has magnitude greater than one $\left(\left|m_{i}\right|>1\right)$.

One-delay control law implies that the duty cycle $d_{k}$ depends on state variables in the instant $(k-1) T$, i.e., $d_{k}=c_{1} x_{1}((k-1) T)+c_{2} x_{2}((k-1) T)+c_{3}$. Two additional state variables can be defined $x_{3}(k T)=x_{1}((k-1) T)$ and $x_{4}(k T)=x_{2}((k-1) T)$. Therefore, $d_{k}=c_{1} x_{3}(k T)+$ $c_{2} x_{4}(k T)+c_{3}$.

In this case, Poincaré map (30) can be written as equation (33).

$$
\begin{array}{lc}
x_{1}((k+1) T)=f_{1}\left(x_{1}(k T), x_{2}(k T), x_{3}(k T), x_{4}(k T)\right) \\
x_{2}((k+1) T)=f_{2}\left(x_{1}(k T), x_{2}(k T), x_{3}(k T), x_{4}(k T)\right) \\
x_{3}((k+1) T)=r & x_{1}(k T) \\
x_{4}((k+1) T)= & x_{2}(k T)
\end{array}
$$

Jacobian matrix of the system with $\tau=1$ can be computed with equation (34).

$$
\mathbf{A}_{n 1}=\left[\begin{array}{cccc}
\frac{\partial f_{1}}{\partial x_{1}(k T)} & \frac{\partial f_{1}}{\partial x_{2}(k T)} & \frac{\partial f_{1}}{\partial x_{3}(k T)} & \frac{\partial f_{1}}{\partial x_{4}(k T)} \\
\frac{\partial f_{2}}{\partial x_{1}(k T)} & \frac{\partial f_{2}}{\partial x_{2}(k T)} & \frac{\partial f_{2}}{\partial x_{3}(k T)} & \frac{\partial f_{2}}{\partial x_{4}(k T)} \\
1 & 0 & 0 & 0 \\
0 & 1 & 0 & 0
\end{array}\right]
$$

The matrix $\mathbf{A}_{n 1}$ should be evaluated in the fixed point $\left(\mathbf{A}_{n 1}=\left(\frac{\partial \mathbf{f}}{\partial \mathbf{x}_{i}}\right)_{\text {F.P. }}\right)$. In this case, we define F.P. as (ref, rref, ref, rref).

Four characteristic multipliers are computed. The 1-periodic orbit is asymptotically stable if the four characteristic multipliers have magnitude less than one.

Characteristic multipliers for delayed PWM control law with $\tau>1$ can be computed following the same procedure. However, The order of Jacobian matrix increases as the delay number grows.

Now, we compute Lyapunov exponents using a numeric routine. This algorithm is based on the definition of Lyapunov exponents. Equation (35) synthesizes this procedure. Poincaré map is used to compute the values of state variables. Jacobian matrix should be known to compute the eigenvalues $q_{i}$ in each iteration $k$.

$$
\lambda_{i}=\operatorname{Lim}_{M \rightarrow \infty}\left\{\frac{1}{M} \sum_{k=0}^{M} \log \left|q_{i}\left(A_{n j}(x(k))\right)\right|\right\}
$$

\subsection{Equivalence between stability methods}

Floquet exponents, Lyapunov exponents and characteristic multipliers are interconnected to each other. Mathematical expressions to relate each approach are synthesized in figure 5 . For 
example, we can compute the Floquet exponents $\left(\mu_{i}\right)$ for any delay and later we can apply the relations $m_{i}=e^{\mu_{i} T}$ and $\lambda_{i}=\mu_{i} T$ to find characteristic multipliers and Lyapunov exponents, respectively.

Figure 6 shows the evolution of Floquet and Lyapunov exponents when the duty cycle is computed without delay and with one delay. The critic values are the equals using any method. Therefore, both methods give the same information.

Figure 7 shows the evolution of Floquet exponents and characteristic multipliers in the complex plane of each representation. In both cases, the parameter $N$ is varied in the range $[0 ; 30]$ with $k_{s}=4.5$ and $\tau=1$. Imaginary axis is the stability limit of the Floquet exponents locus, while unity circle is the stability limit of the characteristic multipliers locus.

\section{Bifurcations in Buck converter with delayed ZAD-FPIC}

In this section, we analyze types of bifurcations in the buck converter controlled with Delayed ZAD-FPIC scheme using the procedure based on Floquet exponents described in previous sections. We transform Floquet exponents in characteristic multipliers using the equivalences shown in figure 5 .

If at least one characteristic multiplier is outside of the unit circle then the system has an unstable fixed point and nonlinear phenomena as quasi-periodicity and chaos could be present. In the boundary, the smooth bifurcations (flip, fold and Neimark-Sacker) are present. The presence of the three smooth bifurcations in the same converter is not common and this fact has not been reported widely in Digital-PWM switched converters [14].

Control parameters $k_{s}$ and $N$ can be varied in $\mathbb{R}$ with the exception of $k_{s}=0$ and $N+1=0$ (because the control law is not defined there). Parameter $\tau$ can be varied in $\mathbb{Z}$. The 3D-parameter space $\left(k_{s}, N, \tau\right)$ is discontinuous due to the discrete delays $(\tau=0,1,2,3$,$) and$ the undefined planes $\left(k_{s}=0\right.$ and $\left.N+1=0\right)$. Figure $8(a)$ shows a representation of the control parameter space.

The two-dimensional plane $\left(k_{s}, N\right)$ can be divided into four regions: region I: $k_{s}>0$ and $N>-1$; region II: $k_{s}<0$ and $N>-1$; region III: $k_{s}<0$ and $N<-1$; and region IV: $k_{s}>0$ and $N<-1$. Fold zones, flip zones and Neimark-Sacker zones can be identified in the control space. The fold bifurcation is an alarm for duty cycle saturation in $d=0 \%$ or $d=100 \%$; the flip bifurcation signals a doubling period and the Neimark-Sacker bifurcation is related to $2 \mathrm{D}$-torus birth.

Computer simulations are given for the purpose of illustration and verification. Next, we present the three bifurcations types in the 3D-parameter space.

\subsection{Flip bifurcations in $\left(k_{s}, N, \tau\right)$ space}

This bifurcation is associated with the appearance of a negative real characteristic multiplier in the unit cycle boundary $\left(m_{i}=-1\right)$. Figure $9(a)$ shows the evolution of characteristic multipliers when $N$ is varied in a positive range for several $k_{S}$ values. 


\subsubsection{Control subspaces}

The flip bifurcations have been detected in the following $\left(k_{s}-N\right)$ plane: Subspace I when $\tau=0$. The flip zone in the plane $\tau=0$ is presented in figure $8(b)$.

\subsubsection{Characteristics near to flip bifurcation}

Before the flip bifurcation the converter has a stable fixed point or T-periodic orbit. After the flip bifurcation the T-periodic orbit is unstable and the converter has a stable 2T-periodic orbit. Successive flip bifurcations and border-collision bifurcations are presented until the chaos formation. More details can be found in [2], [4], [15]. An illustrative example is shown in figure $10(a)$.

\subsection{Fold bifurcations in $\left(k_{s}, N, \tau\right)$ space}

This bifurcation is associated with the appearance of a positive real characteristic multiplier in the unit cycle boundary $\left(m_{i}=1\right)$. Figure $9(b)$ shows the evolution of characteristic multipliers when $N$ is varied in a negative range for several $k_{s}$ and $\tau$ values.

\subsubsection{Control subspaces}

The fold bifurcations have been detected in the following $\left(k_{s}-N\right)$ planes: Subspaces II and IV for (see figure $8(c)$ ).

\subsubsection{Characteristics near to fold bifurcation}

Before the fold bifurcation the converter has two fixed points: one stable and other unstable. The stable fixed point is near to reference value. After the fold bifurcation the converter has not fixed points and the output is saturated. An illustrative case is presented in figure $10(b)$.

\subsection{Neimark-Sacker bifurcations in $\left(k_{s}, N, \tau\right)$ space}

This bifurcation is associated with the appearance of two conjugate complex characteristic multipliers in the unit cycle boundary. Figure 9 (c) shows the evolution of characteristic multipliers when $N$ and $k_{s}$ are varied in positive ranges for $\tau>1$.

\subsubsection{Control subspaces}

The Neimark-Sacker bifurcations have been detected in the following $\left(k_{s}-N\right)$ planes: Subspace III for $\tau=0$ and Subspace I and III for $\tau>0$. The control subspaces with Neimark-Sacker bifurcations are presented in figure $8(d)$.

\subsubsection{Characteristics near to Neimark-Sacker bifurcation}

Before the Neimark-Sacker bifurcation the converter has a stable fixed point or T-periodic orbit. After the Neimark-Sacker bifurcation the converter has quasi-periodic behavior and 2D-torus birth. The bifurcation diagram and the characteristic multipliers in the Neimark-Sacker transition are shown in figure $10(c)$. 


\section{Conclusions and future work}

In this chapter, we have presented a generalized procedure to compute Floquet exponents for any number of delays $(\tau)$ in the control law of PWM switched converters. We have investigated the incidence of delays in a digital-PWM controller based on two novel techniques: Zero Average Dynamics (ZAD) and Fixed-Point Inducting Control (FPIC). Principles of Floquet theory and smooth bifurcation theory were used to define stability regions. The incidence of control parameters in transient response of PWM switched converters will be analyzed in a future work using a similar Floquet-based procedure.

\section{Author details}

John Alexander Taborda

Universidad del Magdalena - Facultad de Ingeniería - Programa de Ingeniería Electrónica - Magma Ingeniería - Santa Marta D.T.C.H., 2121630, Colombia

Fabiola Angulo and Gerard Olivar

Universidad Nacional de Colombia - Sede Manizales - Facultad de Ingeniería y Arquitectura Departamento de Ingeniería Eléctrica, Electrónica y Computación - Percepción y Control Inteligente Bloque Q, Campus La Nubia, Manizales, 170003 - Colombia

\section{References}

[1] Angulo F., "Dynamical analysis of PWM-controlled power electronic converters based on the zero average dynamics (ZAD) strategy", Ph. D. Thesis. Technical University of Catalonia (in Spanish), 2004.

[2] Angulo F., Fossas E. \& Olivar G. "Transition From Periodicity to Chaos in a PWM-Controlled Buck Converter with ZAD strategy", International Journal of Bifurcations and Chaos, 15, 10, 3245-3264, 2005.

[3] Angulo F., Ocampo C., Olivar G. \& Ramos R., Nonlinear and Nonsmooth Dynamics in a DC-DC Buck Convreter: Two experimental set-ups Nonlinear Dynamics, Vol. 46, No. 3, pp. 239-257, 2006.

[4] Angulo F., Olivar G. \& Taborda J.A., "Continuation of periodic orbits in a ZAD-strategy controlled buck converter", Chaos, Solitons and Fractals, 38, 348-363, 2008.

[5] Angulo F., Hoyos F.E. \& Taborda J.A., "Principios de la Estrategia de Control Zero Average Dynamics (ZAD)" (in Spanish). Lambert Academic Publishing, Madrid, España, 2011.

[6] Awrejcewicz J., "Bifurcation and Chaos in Simple Dynamical Systems”. World Scientific, Singapore, 1989.

[7] Awrejcewicz J., "Three routes to chaos in simple sinusoidally driven oscillators”, Journal of Applied Mathematics and Mechanics ZAMM, 71 (2), 1991, 71-79.

[8] Awrejcewicz J., "Numerical analysis of the oscillations of human vocal cords", Nonlinear Dynamics, 2, 1991, 35-52.

[9] Awrejcewicz J., Kudra G. \& Lamarque C-H., "Investigation of triple pendulum with impacts using fundamental solution matrices", International Journal of Bifurcation and Chaos, 14 (12), 2004, 4191-4213. 
[10] Fossas E. \& Zinober A., Adaptive tracking control of nonlinear power converters In Proceedings IFAC Workshop on Adaptation in Control and Signal Processing. Connobio. Italy. pp 264-266, 2001.

[11] Hart D., “Power Electronics”. Prentice Hall, Madrid, España, 2001.

[12] Mohan N., Undeland T. \& Robbins W., "Power Electronics: Converters, applications and design", J. Wiley, 1995.

[13] Taborda J.A., "Bifurcation analysis in second-order systems with PWM based on zero average dynamics (ZAD) strategy.", Master Thesis. National University of Colombia (in Spanish), Manizales, Colombia, 2006.

[14] Taborda J.A., Angulo F. \& Olivar G., "Smooth Bifurcations in 3D-parameter Space of Digital-PWM Switched Converter", In Proceedings of 2nd IEEE Latin American symposium on circuits and Systems - LASCAS 2011. Bogotá. Colombia, 2011.

[15] Taborda J.A., Angulo F. \& Olivar G., "Characterization of chaotic attractors inside band-merging scenario in ZAD-controlled buck converter", Special Issue of 2010 DDays, International Journal of Bifurcations and Chaos, (to appear) . 